\title{
Steady states of a nonlinear elliptic system arising from population dynamics
}

\author{
Ruyun Ma*, Ruipeng Chen and Yanqiong Lu
}

\section{"Correspondence:}

mary@nwnu.edu.cn

Department of Mathematics,

Northwest Normal University,

Lanzhou, 730070, P.R. China

\begin{abstract}
In this paper, we study the existence and multiplicity of coexistence states for an elliptic system modeling two subpopulations of the same species competing for resources. Our results generalize and complement the work of Bouguima et al. (Nonlinear Anal., Real World Appl. 9:1184-1201, 2008). In particular, an open problem proposed by Bouguima et al. is partially solved.
\end{abstract}

MSC: 34B10; 34B18

Keywords: elliptic systems; coexistence states; existence; multiplicity; bifurcation

\section{Introduction}

This paper studies the coexistence states of the nonlinear elliptic system

$$
\begin{cases}-\Delta u=a(x) v-e(x) u-c(x) u(u+v), & x \in \Omega, \\ -\Delta v=b(x) u-f(x) v-d(x) v(u+v), & x \in \Omega, \\ u=v=0, & x \in \partial \Omega,\end{cases}
$$

where $\Omega$ is a bounded regular domain of $\mathbb{R}^{n}(n \geq 1)$, i.e., $\bar{\Omega}$ is an $n$-dimensional compact connected smooth submanifold of $\mathbb{R}^{n}$ with boundary $\partial \Omega$. Throughout the paper, we suppose that $a, b, c, d, e$, and $f$ are positive functions in $C^{\alpha}(\bar{\Omega})$ for a certain $\alpha \in(0,1)$.

System (1.1) arises from population dynamics where it models the steady-state solutions of the corresponding nonlinear evolution problem [1], where $u$ and $v$ represent, respectively, the concentrations of the adult and juvenile populations. The function $a$ gives the rate at which juveniles become adults and as adults give birth to juveniles, the function $b$ corresponds to the birth rate of the population, $e$ and $f$ reflect the result of harvesting a portion of the population (fishing effort for marine population), $c$ and $d$ measure the competition between $u$ and $v$. Both populations are living in the same region $\Omega$, and the boundary conditions in (1.1) may be interpreted as the condition that the populations $u$ and $v$ may not stay on $\partial \Omega$. The Laplacian operator shows the diffusive character of $u$ and $v$ within $\Omega$.

Obviously, system (1.1) can be rewritten as

$$
\begin{cases}\mathfrak{L} U=A(x) U-F(U), & x \in \Omega, \\ U=0, & x \in \partial \Omega,\end{cases}
$$

C 2014 Ma et al.; licensee Springer. This is an Open Access article distributed under the terms of the Creative Commons Attribution License (http://creativecommons.org/licenses/by/4.0), which permits unrestricted use, distribution, and reproduction in any medium, provided the original work is properly credited. 
where $U=(u, v)^{T}, \mathfrak{L}=\operatorname{diag}(-\Delta,-\Delta)$,

$$
A(x)=\left(\begin{array}{cc}
-e(x) & a(x) \\
b(x) & -f(x)
\end{array}\right)
$$

and $F$ the nonlinear term such that $F(U)(x)=\left(\begin{array}{l}c(x) u(u+v) \\ d(x) v(u+v)\end{array}\right)$. In the following, for $q \in L^{\infty}(\Omega)$, we denote by $\underline{q}$ and $\bar{q}$ the essential infimum and supremum of $q$, respectively. Let $\rho_{1}(q)$ be the principal eigenvalue of the linear problem

$$
\begin{cases}-\Delta u+q(x) u=\rho u, & x \in \Omega, \\ u=0, & x \in \partial \Omega,\end{cases}
$$

and $\varphi_{1}(q)$ the principal eigenfunction associated to $\rho_{1}(q)$. Then it is not difficult to check that $\rho_{1}(q)=q+\lambda_{1}$ and $\varphi_{1}(q)=\varphi_{1}$ if $q(\cdot) \equiv q$ is a constant. Here $\left(\lambda_{1}, \varphi_{1}\right)$ is the principal eigen-pair of $-\Delta$ under a homogeneous Dirichlet boundary condition such that $\varphi_{1}(x)>0$, $x \in \Omega$, and

$$
\left\|\varphi_{1}\right\|_{\infty}=\max _{x \in \bar{\Omega}}\left|\varphi_{1}(x)\right|=1
$$

In recent years, system (1.1) has been studied by several authors, see for example, [1-5] and the references listed therein. In [1] and [2], system (1.1) was discussed as a problem in optimal control. Existence and uniqueness results are given in terms of hypotheses which are appropriate for control problems, that is, the coefficients are required to satisfy certain uniform bounds. Brown and Zhang [3] studied system (1.1) subject to Neumann boundary condition, and obtained more precise existence results in terms of the principal eigenvalue of

$$
\begin{cases}\mathfrak{L} U-A(x) U=\mu U, & x \in \Omega, \\ \frac{\partial U}{\partial \mathbf{n}}=0, & x \in \partial \Omega,\end{cases}
$$

where $\mathbf{n}$ is the outward unit normal vector on $\partial \Omega$; see [3, Theorem 3.2]. Recently, Bouguima et al. [4] investigated system (1.1) and showed that it has a positive solution if and only if $\lambda_{1}(\mathfrak{L}-A(x))<0$ via fixed point theory, where $\lambda_{1}(\mathfrak{L}-A(x))$ is the principal eigenvalue of the linear eigenvalue problem

$$
\begin{cases}\mathfrak{L} U-A(x) U=\lambda U, & x \in \Omega, \\ U=0, & x \in \partial \Omega .\end{cases}
$$

We note that $\lambda_{1}(\mathfrak{L}-A(x))$ exists if $a(x)>0, b(x)>0$ for $x \in \Omega$; see Lemma 12 and Corollary 18 in [4] for the details. In addition, implicit function theorem was adopted in [4] to discuss the limit case of system (1.1), where the authors supposed that $a(x)=\rho_{1}(e)+\varepsilon a_{1}(x)$, $b(x)=\rho_{1}(e)+\varepsilon b_{1}(x)$ with $\varepsilon>0$ sufficiently small and $a_{1}, b_{1} \in L_{+}^{\infty}(\Omega)$; the strength of the crowding effect and the competition between $u$ and $v$ are negligible, i.e., $c(x)=\varepsilon c_{1}(x)$, $d(x)=\varepsilon d_{1}(x)$ for $c_{1}, d_{1} \in L_{+}^{\infty}(\Omega)$, and small $\varepsilon>0$; the functions $e$ and $f$ are assumed to have the same effect, that is, $f(x)=e(x)+\varepsilon f_{1}(x)$ with $f_{1} \in L_{+}^{\infty}(\Omega)$ and $\varepsilon>0$ is small enough. Under the above assumptions, Bouguima et al. obtained the following multiplicity results. 
Theorem A [4, Theorem 24] Assume that

$$
\alpha:=\frac{1}{4} \int_{\Omega}\left(a_{1}+b_{1}-f_{1}\right) \varphi_{1}^{2}(e) d x>0, \quad \beta:=\frac{1}{2} \int_{\Omega}\left(c_{1}+d_{1}\right) \varphi_{1}^{3}(e) d x>0 .
$$

Then there exists $\varepsilon_{0}>0$, such that system (1.1) has two nontrivial solutions of the form

$$
\xi_{i}(\varepsilon)=S^{i}(\varepsilon)\left(\varphi_{1}(e), \varphi_{1}(e)\right)+U\left(S^{i}(\varepsilon), \varepsilon\right), \quad i=1,2,
$$

where $S^{1}:\left(-\varepsilon_{0}, \varepsilon_{0}\right) \rightarrow V_{0}$ and $S^{2}:\left(-\varepsilon_{0}, \varepsilon_{0}\right) \rightarrow V_{*}$ are some functions w.r.t. $\varepsilon$, and $V_{0}, V_{*}$ are neighborhoods of 0 and $\frac{\alpha}{\beta}$, respectively. The function $U\left(S^{i}(\varepsilon), \varepsilon\right)(i=1,2)$ appearing in (1.3) is given as in [4, Lemma 23].

By an inspection of the arguments in [4, Section 5], it is easy to see that the second nontrivial solution $\xi_{2}$ obtained in Theorem A is positive and satisfies

$$
(u(\varepsilon), v(\varepsilon)) \rightarrow \frac{\alpha}{\beta}\left(\varphi_{1}(e), \varphi_{1}(e)\right) \quad \text { as } \varepsilon \rightarrow 0,
$$

see also [4, Theorem 2]. However, Bouguima et al. pointed out that the positiveness of the small solution $\xi_{1}$ is not obvious and the question remains open. In this paper, we shall give a partial answer to this open problem.

Our main result can be stated as below.

Theorem 1.1 Let $a_{1}, b_{1}, f_{1}$, and e be positive constants such that $a_{1}+b_{1}-f_{1}>0$. Then the small solution $\xi_{1}$ is positive.

Remark 1.1 We note that the constants $\alpha$ and $\beta$ defined in [4] were wrong, and this mistake is corrected in the statement of Theorem A. Moreover, we would like to point out that under the assumptions (which are similar to the corresponding ones of Theorem A) of Theorem 1.1, the discussions in [4, Section 5] can also be carried out, and thus the positiveness of $\xi_{1}$ remains unclear. However, in our case the positiveness of $\xi_{1}$ could be obtained. Consequently, the open problem proposed by Bouguima et al. [4] is partially solved, and it is the first time to obtain the multiplicity of positive solutions for system (1.1). For other related results on this topic, we refer the readers to [6-8] and the references therein.

\section{Proof of Theorem 1.1}

To obtain the positiveness of $\xi_{1}$, we introduce the following nonlinear elliptic system:

$$
\begin{cases}-\Delta u=\tilde{a} u+\tilde{b} v+f(x, u, v), & x \in \Omega, \\ -\Delta v=\tilde{c} u+\tilde{d} v+g(x, u, v), & x \in \Omega, \\ u=v=0, & x \in \partial \Omega,\end{cases}
$$

where $\tilde{a}, \tilde{b}, \tilde{c}, \tilde{d}$ are real parameters. For a matrix $\tilde{A}=\left(\begin{array}{c}\tilde{a} \\ \tilde{b} \\ \tilde{b}\end{array}\right)$, we denote by $\sigma(\tilde{A})$ the spectrum of $\tilde{A}$. Let $\sigma(-\Delta)$ be the spectrum of $-\Delta$ under a homogeneous Dirichlet boundary condition. We shall also use the notation $\tilde{A}_{0}=\left(\begin{array}{cc}\tilde{a}_{0} & \tilde{b}_{0} \\ \tilde{c}_{0} & \tilde{d}_{0}\end{array}\right)$. Let $\&$ be the closure of the set of nontrivial solutions of system (2.1). We define the Banach space

$$
E=\left\{u \in C^{1+\alpha}(\bar{\Omega}): u=0 \text { on } \partial \Omega\right\}
$$


equipped with the usual norm. Denoting by $\partial / \partial \mathbf{n}$ the outward normal derivative, we introduce

$$
K^{+}:=\left\{u \in E: u>0 \text { in } \Omega, \frac{\partial u}{\partial \mathbf{n}}<0 \text { on } \partial \Omega\right\}, \quad K^{-}=-K^{+} \text {. }
$$

The subsets $K^{+}, K^{-}$, and $K=K^{+} \cup K^{-}$are open in $E$.

Lemma 2.1 [9, Lemma 3.1] Assume that $f$ and $g$ are continuous functions in $\Omega \times \mathbb{R}^{2}$ satisfying the following conditions:

(H1) $f(x, r)$ and $g(x, r)$ are continuous and locally Lipschitz with respect to $r=\left(r_{1}, r_{2}\right) \in \mathbb{R}^{2}$.

(H2) $f(\cdot, r)=o(|r|), g(\cdot, r)=o(|r|)$ at 0 , uniformly in $x \in \bar{\Omega}$.

Suppose $\sigma\left(\tilde{A}_{0}\right) \cap \sigma(-\Delta)=\left\{\lambda_{1}\right\}$ and either $\tilde{b}_{0}\left(\lambda_{1}-\tilde{a}_{0}\right)>0$ or $\tilde{c}_{0}\left(\lambda_{1}-\tilde{d}_{0}\right)>0$. Then there exists a neighborhood $U_{0}$ of $\left(\tilde{A}_{0},(0,0)\right)$, such that if $(A,(u, v)) \in U_{0} \cap \&$ for some matrix $A$ and $(u, v) \neq(0,0)$, then either $u, v \in K^{+}$or $u, v \in K^{-}$.

Proof of Theorem 1.1 We shall consider the system

$$
\begin{cases}-\Delta u=-e u+\left(\lambda_{1}+e\right) v-\varepsilon c_{1}(x) u(u+v), & x \in \Omega, \\ -\Delta v=\left(\lambda_{1}+e\right) u-e v-\varepsilon d_{1}(x) v(u+v), & x \in \Omega, \\ u=v=0, & x \in \partial \Omega .\end{cases}
$$

Let

$$
A_{0}=\left(\begin{array}{cc}
-e & \lambda_{1}+e \\
\lambda_{1}+e & -e
\end{array}\right)
$$

Then it is not hard to check that $A_{0}$ has two eigenvalues $\mu_{1}=\lambda_{1}$ and $\mu_{2}=-\lambda_{1}-2 e$, and therefore $\sigma\left(A_{0}\right) \cap \sigma(-\Delta)=\left\{\lambda_{1}\right\}$. Moreover,

$$
\begin{aligned}
& \tilde{b}_{0}\left(\lambda_{1}-\tilde{a}_{0}\right)=\left(\lambda_{1}+e\right)\left(\lambda_{1}-(-e)\right)=\left(\lambda_{1}+e\right)^{2}>0, \\
& \tilde{c}_{0}\left(\lambda_{1}-\tilde{d}_{0}\right)=\left(\lambda_{1}+e\right)\left(\lambda_{1}-(-e)\right)=\left(\lambda_{1}+e\right)^{2}>0 .
\end{aligned}
$$

Clearly, the functions $f(x, u, v):=-\varepsilon c_{1}(x) u(u+v)$ and $g(x, u, v):=-\varepsilon d_{1}(x) v(u+v)$ satisfy $(\mathrm{H} 1)$ and (H2). Consequently, all of the assumptions of Lemma 2.1 are satisfied.

On the other hand, by Theorem A, we know that the system

$$
\begin{cases}-\Delta u=-e u+\left(\lambda_{1}+e+\varepsilon a_{1}\right) v-\varepsilon c_{1}(x) u(u+v), & x \in \Omega, \\ -\Delta v=\left(\lambda_{1}+e+\varepsilon b_{1}\right) u-\left(e+\varepsilon f_{1}\right) v-\varepsilon d_{1}(x) v(u+v), & x \in \Omega, \\ u=v=0, & x \in \partial \Omega\end{cases}
$$

admits the nontrivial small solution $\xi_{1}$, i.e., the nontrivial solution $\left(A_{\varepsilon},\left(u_{\varepsilon}, v_{\varepsilon}\right)\right)$ with $u_{\varepsilon}$ and $v_{\varepsilon}$ small, where

$$
A_{\varepsilon}=A_{0}+\varepsilon\left(\begin{array}{cc}
0 & a_{1} \\
b_{1} & -f_{1}
\end{array}\right)
$$


In addition, since $\varepsilon>0$ is sufficiently small, it follows from Lemma 2.1 that the nontrivial small solution $\left(A_{\varepsilon},\left(u_{\varepsilon}, v_{\varepsilon}\right)\right)$ lies in the neighborhood $u_{0}$ of $\left(A_{0},(0,0)\right)$, and either $u_{\varepsilon}, v_{\varepsilon} \in$ $K^{+}$or $u_{\varepsilon}, v_{\varepsilon} \in K^{-}$.

We claim that $u_{\varepsilon}, v_{\varepsilon} \in K^{+}$.

Suppose on the contrary that $u_{\varepsilon}, v_{\varepsilon} \in K^{-}$. Then we have

$$
u_{\varepsilon}(x)<0, \quad v_{\varepsilon}(x)<0, \quad x \in \Omega,
$$

and they satisfy

$$
\begin{cases}-\Delta u_{\varepsilon}=-e u_{\varepsilon}+\left(\lambda_{1}+e+\varepsilon a_{1}\right) v_{\varepsilon}-\varepsilon c_{1}(x) u_{\varepsilon}\left(u_{\varepsilon}+v_{\varepsilon}\right), & x \in \Omega, \\ -\Delta v_{\varepsilon}=\left(\lambda_{1}+e+\varepsilon b_{1}\right) u_{\varepsilon}-\left(e+\varepsilon f_{1}\right) v_{\varepsilon}-\varepsilon d_{1}(x) v_{\varepsilon}\left(u_{\varepsilon}+v_{\varepsilon}\right), & x \in \Omega, \\ u_{\varepsilon}=v_{\varepsilon}=0, & x \in \partial \Omega .\end{cases}
$$

Let $A_{\varepsilon}^{T}$ be the transpose of $A_{\varepsilon}$. Then it is easy to verify that $\mathfrak{L}-A_{\varepsilon}^{T}$ is the adjoint operator of $\mathfrak{L}-A_{\varepsilon}$. By the well-known functional analytic techniques (see $[10,11]$ and $[12$, Lemma 2.3]), we know that the principal eigenvalue $\lambda_{1}\left(\mathfrak{L}-A_{\varepsilon}\right)$ of $\mathfrak{L}-A_{\varepsilon}$ is also the principal eigenvalue of $\mathfrak{L}-A_{\varepsilon}^{T}$. In the following, we denote by $\left(\psi_{1}, \psi_{2}\right)^{T}$ the unique eigenfunction of $\mathfrak{L}-A_{\varepsilon}^{T}$ (up a constant multiple) associated to $\lambda_{1}\left(\mathfrak{L}-A_{\varepsilon}\right)$, such that $\psi_{1}>0, \psi_{2}>0$ in $\Omega$. Then $\left(\psi_{1}, \psi_{2}\right)^{T}$ verifies that

$$
\begin{cases}-\Delta \psi_{1}=-e \psi_{1}+\left(\lambda_{1}+e+\varepsilon b_{1}\right) \psi_{2}+\lambda_{1}\left(\mathfrak{L}-A_{\varepsilon}^{T}\right) \psi_{1}, & x \in \Omega, \\ -\Delta \psi_{2}=\left(\lambda_{1}+e+\varepsilon a_{1}\right) \psi_{1}-\left(e+\varepsilon f_{1}\right) \psi_{2}+\lambda_{1}\left(\mathfrak{L}-A_{\varepsilon}^{T}\right) \psi_{2}, & x \in \Omega, \\ \psi_{1}=\psi_{2}=0, & x \in \partial \Omega .\end{cases}
$$

Multiplying the system $(2.5)$ by $\left(\psi_{1}, \psi_{2}\right)^{T}$, the system $(2.6)$ by $\left(u_{\varepsilon}, v_{\varepsilon}\right)^{T}$, integrating over $\Omega$, and subtracting, we can get

$$
\begin{aligned}
\lambda_{1}\left(\mathfrak{L}-A_{\varepsilon}^{T}\right) \cdot \int_{\Omega}\left(\psi_{1} u_{\varepsilon}+\psi_{2} v_{\varepsilon}\right) d x \\
\quad=-\varepsilon \int_{\Omega}\left(c_{1}(x) u_{\varepsilon}\left(u_{\varepsilon}+v_{\varepsilon}\right) \psi_{1}+d_{1}(x) v_{\varepsilon}\left(u_{\varepsilon}+v_{\varepsilon}\right) \psi_{2}\right) d x,
\end{aligned}
$$

which together with (2.4) implies $\lambda_{1}\left(\mathfrak{L}-A_{\varepsilon}^{T}\right)>0$, and hence $\lambda_{1}\left(\mathfrak{L}-A_{\varepsilon}\right)>0$. Consequently, by [4, Corollary 18], we know that system (1.1) has no positive solutions. But this is impossible since (1.1) admits a positive solution $\xi_{2}$.

\section{Related results}

In this section, we shall investigate the effect of death rates and birth rates on the coexistence states. Bouguima et al. [4] established the following existence result.

Theorem B [4, Theorem 20] Assume $f=e+k$ with $k \in \mathbb{R}$. If $\underline{a b}>\rho_{1}(e) \rho_{1}(f)$, then system (1.1) admits at least one positive solution.

Obviously, the assumption $f=e+k, k \in \mathbb{R}$ used in Theorem B is too restrictive, we shall weaken it below. Since $a, b, c, d$, e, and $f$ are $C^{\alpha}(\bar{\Omega})$ continuous positive functions, 
system (1.2) has a principal eigenvalue $\lambda_{1}(\mathfrak{L}-A(x)$ ), which corresponds to a unique (up to a constant multiple) eigenfunction

$$
U_{0}=\left(u_{0}, v_{0}\right)^{T} \in C^{2, \alpha}(\bar{\Omega}) \times C^{2, \alpha}(\bar{\Omega})
$$

satisfying $u_{0}>0, v_{0}>0$ in $\Omega$.

Theorem 3.1 System (1.1) has at least one positive solution provided that

$$
\max \left\{\bar{e}+\lambda_{1}, \bar{f}+\lambda_{1}\right\}<\min \left\{\underline{a} \frac{\int_{\Omega} \varphi_{1}(e) v_{0} d x}{\int_{\Omega} \varphi_{1}(e) u_{0} d x}, \underline{b} \frac{\int_{\Omega} \varphi_{1}(f) u_{0} d x}{\int_{\Omega} \varphi_{1}(f) v_{0} d x}\right\}
$$

Proof By $\left[4\right.$, Corollary 18], to prove the theorem we need only to show that $\lambda_{1}(\mathfrak{L}-A(x))<0$. Recall that $U_{0}=\left(u_{0}, v_{0}\right)^{T}$ satisfies

$$
\begin{cases}-\Delta u_{0}+e(x) u_{0}-a(x) v_{0}=\lambda_{1}(\mathfrak{L}-A(x)) u_{0}, & x \in \Omega, \\ -\Delta v_{0}+f(x) v_{0}-b(x) u_{0}=\lambda_{1}(\mathfrak{L}-A(x)) v_{0}, & x \in \Omega, \\ u_{0}=v_{0}=0, & x \in \partial \Omega .\end{cases}
$$

Multiplying (3.2) with $\left(\begin{array}{c}\varphi_{1}(e) \\ \varphi_{1}(f)\end{array}\right)$, multiplying the system

$$
\begin{cases}-\Delta \varphi_{1}(e)+e(x) \varphi_{1}(e)=\rho_{1}(e) \varphi_{1}(e), & x \in \Omega, \\ -\Delta \varphi_{1}(f)+f(x) \varphi_{1}(f)=\rho_{1}(f) \varphi_{1}(f), & x \in \Omega,\end{cases}
$$

with $\left(u_{0}, v_{0}\right)^{T}$, integrating over $\Omega$, and subtracting, we can obtain

$$
\begin{aligned}
& \lambda_{1}(\mathfrak{L}-A(x))\left(\int_{\Omega} \varphi_{1}(e) u_{0} d x+\int_{\Omega} \varphi_{1}(f) v_{0} d x\right) \\
& =\rho_{1}(e) \int_{\Omega} \varphi_{1}(e) u_{0} d x-\int_{\Omega} a(x) \varphi_{1}(e) v_{0} d x+\rho_{1}(f) \int_{\Omega} \varphi_{1}(f) v_{0} d x-\int_{\Omega} b(x) \varphi_{1}(f) u_{0} d x \\
& \leq\left(\bar{e}+\lambda_{1}\right) \int_{\Omega} \varphi_{1}(e) u_{0} d x-\underline{a} \int_{\Omega} \varphi_{1}(e) v_{0} d x \\
& \quad+\left(\bar{f}+\lambda_{1}\right) \int_{\Omega} \varphi_{1}(f) v_{0} d x-\underline{b} \int_{\Omega} \varphi_{1}(f) u_{0} d x
\end{aligned}
$$

which together with (3.1) implies $\lambda_{1}(\mathfrak{L}-A(x))<0$.

Remark 3.1 Theorem B was established in the special case $f=e+k, k \in \mathbb{R}$. In such a case, we have $\rho_{1}(f)=\rho_{1}(e)+k$ and $\varphi_{1}(e)=\varphi_{1}(f)$. By (3.1) we can easily get

$$
\underline{a} \frac{\int_{\Omega} v_{0} \varphi_{1}(e) d x}{\int_{\Omega} u_{0} \varphi_{1}(e) d x}>\bar{e}+\lambda_{1} \geq \rho_{1}(e), \quad \underline{b} \frac{\int_{\Omega} u_{0} \varphi_{1}(e) d x}{\int_{\Omega} \nu_{0} \varphi_{1}(e) d x}>\bar{f}+\lambda_{1} \geq \rho_{1}(f),
$$

and therefore $\underline{a b}>\rho_{1}(e) \rho_{1}(f)$, which is just the crucial condition used in Theorem B. Although (3.1) seems to be stronger than the assumption of Theorem $B$, the restrictive con$\operatorname{dition} f=e+k, k \in \mathbb{R}$ is weakened, and Theorem 3.1 is applicable to more general classes of $f$ and $e$ rather than $f=e+k, k \in \mathbb{R}$. 
Competing interests

The authors declare that they have no competing interests.

\section{Authors' contributions}

RM and RC completed the main study, carried out the results of this article and drafted the manuscript. YL checked the proofs and verified the calculation. All the authors read and approved the final manuscript.

\section{Acknowledgements}

The authors are very grateful to the anonymous referees for their valuable suggestions. This work was supported by the NSFC (No. 11361054), SRFDP (No. 20126203110004), Gansu provincial National Science Foundation of China (No. 1208RJZA258).

Received: 24 March 2014 Accepted: 16 July 2014 Published online: 24 September 2014

\section{References}

1. Arino, O, Montero, JA: Optimal control of a nonlinear elliptic population system. Proc. Edinb. Math. Soc. 116, 225-241 (2000)

2. Canada, A, Magal, P, Montero, JA: Optimal control of harvesting in a nonlinear elliptic system arising from population dynamics. J. Math. Anal. Appl. 254, 571-586 (2001)

3. Brown, KJ, Zhang, Y: On a system of reaction-diffusion equations describing a population with two age groups. J. Math. Anal. Appl. 282, 444-452 (2003)

4. Bouguima, SM, Fekih, S, Hennaoui, W: Spatial structure in a juvenile-adult model. Nonlinear Anal., Real World Appl. 9, 1184-1201 (2008)

5. Henaoui, O: An elliptic system modeling two subpopulations. Nonlinear Anal., Real World Appl. 13, 2447-2458 (2012)

6. Bouguima, SM, Mehlia, FZ: Asymptotic behavior of an age-structured population model with diffusion. J. Appl. Anal. Comput. 2, 351-362 (2012)

7. Hei, L: Existence and uniqueness of coexistence states for an elliptic system coupled in the linear part. Nonlinear Anal., Real World Appl. 5, 881-893 (2004)

8. Hei, L, Wu, J: Existence and stability of positive solutions for an elliptic cooperative system. Acta Math. Sin. Engl. Ser 21, 1113-1120 (2005)

9. Fleckinger, J, Gil, RPS: Bifurcation for an elliptic system coupled in the linear part. Nonlinear Anal. 37, 13-30 (1999)

10. Sweers, G: Strong positivity in $C(\bar{\Omega})$ for elliptic systems. Math. Z. 209, 251-271 (1992)

11. Kato, T: Perturbation Theory for Linear Operators. Classics in Mathematics. Springer, Berlin (1995). Reprint of the 1980 edition

12. $\mathrm{Wu}, \mathrm{B}, \mathrm{Cui}, \mathrm{R}$ : Existence, uniqueness and stability of positive solutions to a general sublinear elliptic systems. Bound. Value Probl. 74, 1-14 (2013)

\section{Submit your manuscript to a SpringerOpen ${ }^{\circ}$ journal and benefit from:}

- Convenient online submission

- Rigorous peer review

- Immediate publication on acceptance

- Open access: articles freely available online

- High visibility within the field

- Retaining the copyright to your article 